\title{
Intermittent Hemodialysis Low Intensity vs. on Line Hemodiafiltration in Critically Ill Patients with Sepsis and Acute Kidney Injury. Choosing the Best Treatment in a Developing Country
}

Jiménez Darío ${ }^{1 *}$, Guañuna Manuel ${ }^{1}$, Aguilar Ana ${ }^{1}$, Morales Miguel ${ }^{1}$, Jiménez Fernando ${ }^{1,3}$, Dueñas Anunciata ${ }^{1}$, Castillo Marìa ${ }^{1}$, González Rodrigo ${ }^{1}$, Paredes Gustavo $^{2}$, Pazos Ernesto ${ }^{2}$ and Trujillo Freddy ${ }^{2}$

${ }^{1}$ Clinical Research Department, DIALNEF, USA

${ }^{2}$ Intensive Care Unit, Enrique Garcés Hospital, Quito, USA

${ }^{3}$ International University of Ecuador, Quito, USA

*Corresponding author: Jiménez Darío, Clinical Research Department, DIALNEF, Quito, Pichincha 170118, USA, Tel: 593992773176/59322903361; E-mail: djdariojac@yahoo.com

Received Date: June 29, 2017; Accepted Date: August 02, 2017; Published Date: August 09, 2017

Copyright: (C) 2017 Darío J, et al. This is an open-access article distributed under the terms of the Creative Commons Attribution License, which permits unrestricted use, distribution, and reproduction in any medium, provided the original author and source are credited.

\section{Abstract}

Introduction: Acute kidney injury is a serious condition in critically ill patients with sepsis, in Ecuador the prevalence is about of $10 \%$ and has high undocumented mortality rate. The type of renal replacement therapy used is also very discussed in countries that have several treatment options. Many developing regions don't have continuous treatments available for these patients.

Objective: The aim of the study was to demonstrate advantages of treatment with on-line hemodiafiltration (own scheme) versus intermittent hemodialysis high flux low intensity in patients with sepsis and acute kidney injury. Primary outcomes evaluated were: mortality, vasoactive drugs, mechanical ventilation dependence and permanence at intensive care unit.

Results: Cohort and tracing study in patients with sepsis and acute kidney injury. Two groups undergoing treatment for intermittent hemodialysis high flux low intensity (Group A: $n$ 35) vs. on line hemodiafiltration (Group B: n 30). General mortality was $49.2 \%$, (Group A: $60 \%$ vs. group B: $36.6 \% p=0.061$ ). APACHE 2 and SOFA index in both groups were similar ( $p=0.26$ and 0.98 respectively). Time intensive care unit stay (GA: 16.7; GB: 9.9 $p=0.044)$, vasoactive drugs dependence (GA: 9; GB: $4.1 \mathrm{p}=0.084)$, mechanical ventilation dependence (GA: 11.2; GB: 6.5 $p=0.12)$.

Conclusion: On line hemodiafiltration showed benefits statiscally significative in intensive care unit stay, in relation to vasoactive drugs, mechanical ventilation dependence showed beneficies but weren't significatives. The mortality wasn't statistically significative. Results showed advantages in on line hemodiafiltration with our own scheme in critically ill patients with sepsis.

Keywords: On-line hemodiafiltration; Acute kidney injury; Critically ill patients

\section{Introduction}

Acute kidney injury (AKI) is a common clinical condition caused by many etiologies and associated with poor results, including high rate of mortality in the intensive care unit (ICU) and has regional dependence [1], reports vary from $18 \%$ to $67 \%$ according to several authors, the incidence has been increasing, depending on the criteria used in different studies to categorize renal failure $[2,3]$.

The author's opinion is that AKI in critically ill patients has increment of mortality rates determined by the duration and severity of kidney damage, patient's haemodynamic condition, starting time and type of renal replacement therapy (RRT) used.

Actually it is difficult to determine AKI's incidence because of the multiple definitions used, the site where they occur (community, hospitalization, ICU) and the diversity of clinical presentation (sepsis, haemodynamic compromise, concomitant heart failure, intravenous contrast and other toxics, age, race, etc.). A meta-analysis published in June 2013, showed high heterogeneity of AKI incidence with imbalances between the northern and southern hemispheres and between socioeconomic statuses of countries [4]. A comprehensive study conducted by the SLANH, determined that AKI has a bimodal pattern; in urban areas, the behavior is similar to high-income countries; while in the rural area observed a different pattern aimed young, healthy patients influenced by environmental and socioeconomic cultural conditions $[5,6]$.

In Latin America the majority of patients are treated with support of the public health system. The intermittent hemodialysis is the treatment most frequently used in all units, also extended slow dialysis (SLED) was developed $40 \%$, and only $23 \%$ provided continuous renal replacement therapy (CRRT); Peritoneal Dialysis is available only in one-third of the units, and is used in less than $20 \%$ in patients with AKI [7]. In Ecuador CRRT is not available. A prospective study carried out in Quito-Ecuador analyzed 2279 patients admitted at ICU. 210 
Citation: Darío J, Manuel G, Ana A, Miguel M, Fernando J, et al. (2017) Intermittent Hemodialysis Low Intensity vs. on Line Hemodiafiltration in Critically III Patients with Sepsis and Acute Kidney Injury. Choosing the Best Treatment in a Developing Country. J Nephrol Ther 7: 299. doi:10.4172/2161-0959.1000299

Page 2 of 5

(9.2\%) met the criteria of AKI, the main cause was sepsis, of 210 patients 160 (76\%) required RRT [8].

AKI is an independent mortality factor at six month follow-up; the reported mortality varies considerably from $20 \%$ to $75 \%$, depending on the definitions used for AKI and studied clinical subgroups [9]. Sepsis is one of the leading causes of mortality and morbidity in the world, with a tendency towards a gradual increase due to the advanced age of the population and their comorbidities, conditions that raises the incidence and severity of presentation [10].

The development of AKI during a septic process increases morbidity, mortality and has a significant effect on the function of multiple organs; moreover, it is associated with prolonged stay in the ICU and elevation in the consumption of resources in health systems [11]. AKI is diagnosed in approximately two thirds of patients in the first 24 hours of their admission to ICU $[12,13]$, RRT is necessary in $37 \%$ of these patients, around $60 \%$ fully recover kidney function [14].

There is a long debate about the time to start, and the ideal treatment for RRT in critically ill patients with sepsis, considering that the majority have multiorgan dysfunction and hemodynamic instability [8]. Controversies begin since the time of treatment's onset, dose prescription, mode and mechanism for solute's remotion (convection, diffusion, adsorption, or mixed), and about convection therapies there are discussion regarding the replacement volume, although some studies recommend larger volumes of replacement as beneficial [15], these results are not conclusive in other studies [16-18]. ADQI (Acute Dialysis Quality Initiative) recommended the use of high doses of replacement particularly in septic patients [19].

Also there are controversial results when comparing mortality rates between intermittent haemodialysis low intensity (IHD-LI) and intermittent hemodialysis high intensity (IHD-HI) and finally against continuous veno venous hemofiltration $(\mathrm{CVVH})$ [20-25]. In a multicenter, randomized, controlled study was compared the best therapeutic haemodialysis scheme, the aim was to measure mortality at 60 days, results didn't find advantages between intense haemodialysis ( ix sessions a week HD) versus IHD-LI (three times a week for a Kt/V target of 1.2 to 1.4 per session). The study was not conclusive, therefore this reinforced IHD-LI as the therapeutic option of dialysis in patients with AKI admitted to intensive care [8]. There is a scientific fundament for use convection techniques explained on Ronco, Honoré and Alexander's theories [26].

On line hemodiafiltration (OL-HDF) is a mixt technique that combine a standard haemodialysis diffusive transport with a significant amount of convective transport, thus provides a greater clearance of medium and large molecular-size, which are difficult to remove by diffusion alone [27-29]. This technique requires a biocompatible high flux and permeability membranes, as well as precise machines with ultrafiltration control and ultrapure dialysate fluid for replacement. There is a high economic impact in OL - HDF implementation, so it is necessary to know the real benefits for applying [30]. This dialysis technique has been used in renal chronic patients in whom large and medium-size molecules remotion ameliorates chronic complications in hemodialysis patients and decrease mortality [31-33]. Currently by the convective properties, entrapment and elimination of proinflammatory molecules has begun to be used in patients with AKI $[34,35]$.

The hypothesis was that OL-HDF reduces sepsis inflammatory process, keeps on a better hemodynamic status during the treatment, and decreases the directly or indirectly involved factors in critically ill patient death.

In Ecuador there is not available CRRT, so that patients are treated with IHD-LI or IHD-HI or intermittent OL-HDF and there is not a comparative study about benefits between the procedures, so authors decided to develop this study in order to consolidate and choose the best procedure adapted to developing countries.

\section{Materials and Methods}

A multicenter, prospective and comparative study between two renal replacement therapy schemes was conducted to analyze their influence on the primary outcomes. The severity of sepsis was assessed using APACHE II and SOFA scales.

\section{Primary outcome}

All causes of mortality, vasoactive drugs and mechanical ventilation time dependence, length of stay in the ICU of patients treated with two RRT schemes.

\section{Patients}

Inclusion criteria: All patients aged 16 years or more, with sepsis and AKI attended by DIALNEF group, whom belonged to different public and private institutions in Quito-Ecuador, underwent renal replacement therapy, following the author's own schemes. AKI was defined according to the AKIN (Acute Kidney Injury Network) criteria [22].

Exclusion criteria: Patients at intensive care units without sepsis's criteria, patients with hepatitis B, hepatitis C or HIV (DIALNEF don't have equipment for seropositive patients), cases transferred to another ICU, patients with chronic renal failure previously established with acute exacerbation, or with chronic renal disease in RRT, and cases without properly recorded data in medical expedient.

\section{Methods}

Patients were assigned on two groups according the treatment regimen available in different institutions. Group A (GA): Patients who received IHD-LI, the first three sessions were daily with an increasing time of 30 minutes from 150 minutes until 210 minutes, with an increasing blood flow pump (QB) of $50 \mathrm{ml} / \mathrm{min}$ from 250 to 400 $\mathrm{ml} / \mathrm{min}$ and dialysate flow (QD) of $500 \mathrm{ml} / \mathrm{min}$. Since the fourth treatment session patients received standard therapy of 210 minutes with QB $400 \mathrm{ml} / \mathrm{min}$, and QD $500 \mathrm{ml} / \mathrm{min}$ every 48 hours.

Group B (GB): Patients who received OL-HDF, the first three sessions were daily with mixed replacement (pre and post dilutional) therapy according the following scheme:

First session: 180 minutes QB $350 \mathrm{ml} / \mathrm{min}$ QD $800 \mathrm{ml} / \mathrm{min}$, volume replacement $84 \mathrm{ml} / \mathrm{min}$. Second session: 210 minutes QB $400 \mathrm{ml} / \mathrm{min}$ QD $800 \mathrm{ml} / \mathrm{min}$, volume replacement $100 \mathrm{ml} / \mathrm{min}$. Third session: 240 minutes QB $400 \mathrm{ml} / \mathrm{min}$ QD $800 \mathrm{ml} / \mathrm{min}$, volume replacement $120 \mathrm{ml} /$ min. Since the fourth session the patients received the same RRT method that consisting on IHD-LI in sessions of 240 minutes, QB 400 $\mathrm{ml} / \mathrm{min}$, QD $500 \mathrm{ml} / \mathrm{min}$. Treatments were developed with Fresenius $4008 \mathrm{~S}$ machine with on line hemodiafiltration module, polysulfone filters high flux were used in IHD-LI (TORAY sulfone $2.1 \mathrm{~m}^{2}$ surface) and high flux cartridge in OL-HDF (Fresenius FX 1000). 
Citation: Darío J, Manuel G, Ana A, Miguel M, Fernando J, et al. (2017) Intermittent Hemodialysis Low Intensity vs. on Line Hemodiafiltration in Critically III Patients with Sepsis and Acute Kidney Injury. Choosing the Best Treatment in a Developing Country. J Nephrol Ther 7: 299. doi:10.4172/2161-0959.1000299

Page 3 of 5

\section{Statistical analysis}

Data were analyzed with Statistical Package for the Social Sciences (SPSS) operative program 24.0 versions. Continuous variables were described in terms of means, medians, standard deviation and ranges. For samples with normal criteria and homoscedasticity was applied student's T-test. For comorbidity and stockings comparison that did not meet normal criteria Mann-Whitney nonparametric test was applied. Odds ratio (OR) was used to estimate risk of mortality in the two study groups.

\section{Results}

95 patients were admitted to the study. 65 fulfilled all criteria and requirements for the analysis. Treatment modality was $53.8 \%$ (n: 35$)$ in IHD-LI, and 46.2\% (n: 30) in OL-HDF. About sex distribution 56.9\% were male and $43.1 \%$ female. Ages range were from 16 to 87 years, media was in GA 60 and GB 57 and SD 19.2 and 17.3 respectively $(\mathrm{p}=0.36)$.

Severity indicators, which have a direct effect on mortality, showed similarity in both groups. The group's characteristics are summarized in Table 1.

\begin{tabular}{|c|c|c|}
\hline Variable & GA (IHD-LI) & GB (OL-HDF) \\
\hline \multicolumn{3}{|c|}{ Sex: $n$, percentage } \\
\hline Male & $19(54.2 \%)$ & $18(60 \%)$ \\
\hline Female & $16(45.8 \%)$ & $12(40 \%)$ \\
\hline \multicolumn{3}{|c|}{ Severity indicators (media) } \\
\hline SOFA & 12.6 & 12.6 \\
\hline APACHE 2 & 24.4 & 26.8 \\
\hline
\end{tabular}

Table 1: Study group's characteristics.

Overall mortality was $49.2 \%$, despite the important differences in both groups, GA: $60 \%$ and GB: $36.6 \%$, it wasn't statistically significative (Chi: 3.51; p=0.061). Odds: GA: 1.5 -GB: 0.58; Risk Absolut Reduction (RAR): GA: 0.60 (CI: 0.44-0.76); GB: 0.37 (CI: 0.19-0.54) OR: 0.39; Number needed to treatment (NNT): 4.28.

The ICU stay dependence average was: GA: 16.7 vs. GB: 9.97 ( $\mathrm{p}=0,044$; CI: $-13.4-0.17$ ), average need for vasoactive drugs was 6.92 days in all subjects (GA: 9.06 GB: $4.4 \mathrm{p}=0.084 \mathrm{CI}:-9.88-0.63$ ), Mechanical Ventilation dependence was GA: 11.23 vs. GB: $6.50 \mathrm{p}=0.12$ (ci: $-10.8-1.36$.

\begin{tabular}{|l|l|l|l|}
\hline Variable & GA & GB & P \\
\hline Day of vasoactive (day) & 5.57 & 2.53 & 0.049 \\
\hline $\begin{array}{l}\text { Mechanic ventilation } \\
\text { (day) }\end{array}$ & 7.93 & 3.74 & 0.159 \\
\hline ICU time stay (day) & 18.5 & 9.11 & 0.031 \\
\hline
\end{tabular}

Table 2: Principal outcomes in discharged alive patients.

Patients discharged alive from ICU showed the next results: ICU stay in GA: 18.5 vs. GB: 9.11 ( $\mathrm{p}=0.031 \mathrm{CI}$ : $-17.89-0.89$ ); days of treatment with vasoactive drugs: GA: 5.57 vs. GB: 2.53 ( $\mathrm{p}=0.049$
CI:-6.071 -0.19). Mechanical ventilation dependence in GA was 7.93 days and in GB 3.74 days, ( $\mathrm{p}=0.159$ CI:-1.01 - 1.73) (Table 2).

About dialysis sessions in patients discharged alive from ICU, in GA the mean was 7.55 sessions and in GB 5.25, $\mathrm{p}=0.190$. In relation to days needing RRT was 11 days for GA and 7.2 GB p=0.242.

\section{Discussion}

The development of AKI during a septic process increases morbidity, mortality and has a significant effect on the function of multiple organs; moreover, it is associated with prolonged stay in the ICU and elevation in the consumption of resources in health systems [11].

AKI is a serious clinical condition in critically ill septic patients. In this study patients with RRT showed high mortality (49.2\%), similar to those reported in other studies where it reaches up to $70 \%$ [36]. Average age, prevalence in males and severity at admission to ICU are comparable to that reported in similar studies in Spain and AustraliaNew Zealand [37-39]. The severity grade at admission to ICU (APACHE, SOFA score) showed no statistically significant differences between groups, which eliminated an important bias for mortality when comparing both types of RRT.

OL-HDF shows greater benefits than IHD-LI in relation to dependence of vasoactive drugs days, permanence on mechanical ventilation and ICU stay, that has a direct impact on associated comorbidity and mortality of this kind of patients, as well as the optimization of sanitary resources, despite there was not statistically significant difference in mortality p=0.061; OR: 2.3 (IC 0.737-7.197).

OL-HDF as a convective technique reduces the molecules pro inflammatory serum levels (ages, IL-6, IL-10, TNFa all of them related to T helper type I response) 39, which improves the septic process and reduce mortality rates in this kind of patients, this is why it has begun to be used in patients with AKI $[34,35]$.

These two modalities of RRT are easy to perform and are available in our environment, even though OL-HDF is more expensive (400 USD vs. 180 USD per session), but when we analyze the results of ICU stay, this technique is more kindly cost/benefit, considering the value saving of stay in ICU (1500 USD per day).

In the OL-HDF group, mortality average observed was $36.6 \%$ vs. $60 \%$ in IHD-LI group and although it was not statistically significant, provides an OR of 0.39 in favor of the first one.

In Ecuador there is not available CRRT, so that patients are treated with IHD-LI or IHD-HI or intermittent OL-HDF and there is not a comparative study about benefits between the procedures, so authors decided to develop this study in order to consolidate and choose the best procedure adapted to developing countries.

This kind of study should be performed in a bigger group of patients, with the aim to corroborate the results obtained and recommended as an initial therapy in septic patients with AKI as a cost-benefit advantage.

\section{Conclusion}

Relative to results of this paper OL-HDF showed to be better than IHD-LI in many aspects even though there was no statistically significant difference in mortality, allowing us to recommend as first choice OL-HDF with this scheme of treatment proposed for critically 
Citation: Darío J, Manuel G, Ana A, Miguel M, Fernando J, et al. (2017) Intermittent Hemodialysis Low Intensity vs. on Line Hemodiafiltration in Critically III Patients with Sepsis and Acute Kidney Injury. Choosing the Best Treatment in a Developing Country. J Nephrol Ther 7: 299. doi:10.4172/2161-0959.1000299

Page 4 of 5

ill septic patients. This modality of RRT demonstrated benefits about the permanence on mechanical ventilation and need of vasoactive drugs and has an additional benefit when compare cost and sanitary resources with that of the proceeding.

\section{Limitations}

Number of patients who have studied under this type of treatment was too little, it is recommended to extend this study in order to assess possible better becomes of OL-HDF in terms of mortality. Existed international studies that compare IHD-LI vs. CRRT but not to OLHDF daily.

\section{Acknowledgments}

To the DIALNEF group for the dedication to critical patients care with kidney problems and interest to maintain cutting edge technology and support clinical research for the welfare and progress of our society.

\section{References}

1. Cerda J, Bagga A, Kher V, Chakravarthi RM (2008) The contrasting characteristics of acute kidney injury in developed and developing countries. Nat Clin Pract Nephrol 4: 138-153.

2. Uchino S, Kellum JA, Bellomo R, Doig GS, Morimatsu H, et al. (2005) Acute renal failure in critically ill patients: a multinational, multicenter study. JAMA 294: 813-818.

3. Lameire N, Van Biesen W, Vanholder R (2005) Acute renal failure. Lancet 365: 417-430.

4. Susantitaphong P, Cruz DN, Cerda J, Abulfaraj M, Alqahtani F, et al. (2013) World incidence of AKI: A meta-analysis. Clin J Am Soc Nephrol 8: 1482-1493.

5. Daher EF, Lima RS, Silva Júnior GB, Silva EC, Karbage NN, et al. (2010) Clinical presentation of leptospirosis: a retrospective study of 201 patients in a metropolitan city of Brazil. Braz J Infect Dis 14: 3-10.

6. Lombardi R, Yu L, Younes-Ibrahim M, Schor N, Burdmann EA (2008) Epidemiology of acute kidney injury in Latin America. Semin Nephrol 28: 320-329.

7. Lombardi R, Rosa-Diez G, Ferreiro A, Greloni G, Yu L, et al. (2014) Acute Kidney Injury in Latin America: A view on renal replacement therapy resources. Nephrol Dial Transplant 29: 1369-1376.

8. Jiménez D, Morales M, Santacruz C (2013) Acute renal injury (AKI) in intensive care unit (ICU): results with Low Intensity Intermittent Hemodialysis (HDIBI) in Quito-Ecuador. Scientific journal of the Ecuadorian Society of Nephrology 1: 15-24.

9. Bihorac A, Yavas S (2009) Long-term risk of mortality and Acute Kidney Injury during hospitalization after major surgery. Ann Surg 249: 851-858.

10. Zhang Z (2015) Biomarkers, diagnosis and management of sepsisinduced acute kidney injury: a narrative review. Heart Lung Vessel 7: 64-73.

11. Zarjou A, Agarwal A (2011) Sepsis and acute kidney injury. J Am Soc Nephrol 22: 999-1006.

12. Piccinni P, Cruz DN, Gramaticopolo S, Garzotto F, Dal Santo M, et al. (2011) Prospective multicenter study on epidemiology of acute kidney injury in the ICU: A critical care nephrology Italian collaborative effort (NEFROINT). Minerva Anestesiol 77: 1072-1083.

13. Singbartl K, Kellum JA (2012) AKI in the ICU: Definition, epidemiology, risk stratification, and outcomes. Kidney Int 81: 819-825.

14. Eswarappa M, Gireesh MS, Ravi V, Kumar D, Dev G (2014) Spectrum of acute kidney injury in critically ill patients: A single center study from South India. Indian J Nephrol 24: 280-285.

15. Ronco C, Bellomo R, Homel P, Brendolan A, Dan M, et al. (2000) Effects of different doses in continuous veno-venous haemofiltration on outcomes of acute renal failure: a prospective randomised trial. Lancet 356: 26-30.

16. VA/NIH Acute Renal Failure Trial Network, Palevsky PM, Zhang JH, O 'Connor TZ, Chertow GM, et al. (2008) Intensity of renal support in critically ill patients with acute kidney injury. N Engl J Med 359: 7-20.

17. Tolwani AJ, Campbell RC, Stofan BS, Lai KR, Oster RA, et al. (2008) Standard versus high-dose CVVHDF for ICU-related acute renal failure. J Am Soc Nephrol 19: 1233-1238.

18. Tonelli M, Manns B, Feller-Kopman D (2002) Acute renal failure in the Intensive Care Unit: A systematic review of the impact of dialytic modality on mortality and renal recovery. Am J Kidney Dis 40: 875-885.

19. Kellum JA, Bellomo R, Ronco C, Metha R, Clark W, et al. (2005) The 3rd International Consensus Conference of the Acute Dialysis Quality Initiative (ADQI). Int J Artif Organs 28: 441-444.

20. Vesconi S, Cruz D, Ronco C (2009) Dose response multicentre international collaborative Initiative (DO- RE-MI Study Group). Delivered dose of renal replacement therapy and mortality in critically ill patients with acute kidney injury. Critical Care 13: R57.

21. Cho KC, Himmelfarb J, Paganini E, Ikizler TA, Soroko SH, et al. (2006) Survival by dialysis modality in critically ill patients with acute kidney injury. J Am Soc Nephrol 17: 3132-3138.

22. Mehta RL, Kellum JA, Shah SV, Molitoris BA, Ronco C, et al. (2007) Acute kidney injury network: Report of an initiative to improve outcomes in acute kidney injury. Crit Care 11: R31.

23. Alcazar R, De la Torre M, Santana H (2009) Progress in acute renal failure in 2008. Nefrologia 29: 82-87.

24. Ronco C, Zaccaria R, De Backer D, Kellum J, Taccone F, et al. (2015) Renal replacement therapy in acute kidney injury: Controversy and consensus. Critical Care 19: 146.

25. Neuenfeldt T, Hopf HB (2013) Sustained low-efficiency dialysis in an interdisciplinary intensive care unit: A 5-year cost-benefit analysis. Revista Colombiana de Anestesiologia 41: 88-96.

26. Romero C, Downey P, Hernández G (2010) High volume hemofiltration in septic shock. Medicina Intensiva 34: 345-352.

27. Kanter JJ, Puerta M, Perez R, Lopez J, Jofré R, et al. (2008) Sequential line hemodiafiltration (HDF-OL-S): A new therapeutic option. Nefrologia 28: 433-438.

28. Mercadal L, Franck JE, Metzger M, Urena Torres P, de Cornelissen F, et al. (2016) Hemodiafiltration versus hemodialysis and survival in patients with ESRD: The French renal epidemiology and information network (REIN) registry. Am J Kidney Dis 68: 247-255.

29. Wang A, Ninomiya T, Al-Kahwa A, Perkovic V, Gallagher M, et al. (2014) Effect of hemodiafiltration or hemofiltration compared with hemodialysis on mortality and cardiovascular disease in chronic kidney failure: A systematic review and meta-analysis of randomized trials. Am J Kidney Dis 63: 968-978.

30. Ronco C, Canaud B, Aljama P (2007) Hemodiafiltration. Dial Traspl 28: 177.

31. Liabeuf S, Neirynck N, Drueke T, Vanholder R, Massy Z (2014) Clinical studies and chronic kidney disease: What did we learn recently? Semin Nephrol 34: 164-179.

32. Ok E, Asci G, Toz H, Ok ES, Kircelli F, et al. (2013) Mortality and cardiovascular events in online haemodiafiltration (OL-HDF) compared with high-flux dialysis: Results from the Turkish OL-HDF Study. Nephrol Dial Transplant 28: 192-202.

33. National Kidney Foundation (2015) KDOQI clinical practice guideline for hemodialysis adequacy: 2015 update. Am J Kidney Dis 66: 884-930.

34. Farrington K, Davenport A (2013) The ESHOL study: Hemodiafiltration improves survival-but how? Kidney Int 83: 979-981.

35. Maduell F, Moreso F, Mora-Macia J, Pons M, Ramos R, et al. (2015) Reanalysis of the ESHOL study: All-cause mortality considering competition and time-dependent risks for renal transplantation. Nefrologia 36: 156-163.

36. Poukkanen M, Vaara S, Reinikainen M, Selander T, Nisula S, et al. (2015) Predicting one-year mortality of critically ill patients with early acute 
Citation: Darío J, Manuel G, Ana A, Miguel M, Fernando J, et al. (2017) Intermittent Hemodialysis Low Intensity vs. on Line Hemodiafiltration in Critically III Patients with Sepsis and Acute Kidney Injury. Choosing the Best Treatment in a Developing Country. J Nephrol Ther 7: 299. doi:10.4172/2161-0959.1000299

Page 5 of 5

kidney injury: data from the prospective multicenter FINNAKI study. Critical Care 19: 125.

37. Azkarate I, Choperena G, Salas E, Sebastián R, Lara G, et al. (2015) Epidemiology and prognostic factors of severe sepsis / septic shock. Six years of evolution. Medicina Intensiva 40: 18-25.

38. Kaukonen K, Bailey M, Suzuki S, Pilcher D, Bellomo R (2014) Mortality related to severe sepsis and septic shock among critically ill patients in Australia and New Zealand, 2000-2012. JAMA 311: 1308-1316.
39. Rama I, Llaudó I, Fontova P, Cerezo G, Soto C, et al. (2016) Online hemodiafiltration improves inflammatory state in dialysis patients: A longitudinal study. PLOS ONE 11: e0164969. 